\title{
4
}

\section{Sensorless Torque/Force Control}

\author{
Islam S. M. Khalil and Asif Sabanovic \\ Sabanci University \\ Turkey
}

\section{Introduction}

Motion control systems represent a main subsystem for majority of processing systems that can be found in the industrial sector. These systems are concerned with the actuation of all devices in the manufacturing process such as machines, robots, conveyor systems and pick and place mechanisms such that they satisfy certain motion requirements, e.g., the pre specified reference trajectories are followed along with delivering the proper force or torque to the point of interest at which the process occurs. In general, the aim of force/torque control is to impose the desired force on the environment even if the environment has dynamical motion.

The previous motion requirements can be achieved when torque and position/velocity sensors are utilized. However, it is commonly agreed that force/torque sensors have many drawbacks such as (Iida \& Ohnishi, 2003; Katsura et al., 2007):

- Sensor noise: the force/torque sensing process depends on amplifying the strain gauge strain. Therefore, the noise signal is amplified along with the measured signal.

- Narrow bandwidth: due to the previous sensor noise problem, force measurement has to be realized through a low-pass filter. Therefore, the bandwidth of the force sensor is limited by the bandwidth of the sensor noise.

- Complicity and non-collocation: utilization of force/torque sensors add an extra degree of freedom to the control system due to the soft structure of the force sensor, i.e., an energy storage element will exist between the actuated degree of freedom and the end effector in contact with the environment

- Instability: the previous problems cause instability

- The complex electronic setups and their associated wirings with each force or torque sensor embedded to the control system.

The previous drawbacks make the force measurement process undesirable and motivated many researchers over the past few decades to develop force observers to provide alternatives for the problematic force sensing process (Katsura et al., 2007). It was also shown that stable contact between the robot end effector and environment in the presence of force sensing is difficult due to the non-collocation and the soft structure of the force sensor (Li \& Chen, 1998). The bandwidth of the force control using force observers is analyzed and the tradeoffs between stability and response were studied by many researchers to provide a comprehensive guidelines for force observer utilization (Katsura et al., 2008). In addition, the sensorless 
force/torque control techniques found many applications in variety of disciplines such as bilateral teleoperation, microsystems and micro manipulation. Furthermore, the previous force observers can be combined with most of the relevant existing force control techniques such as the hybrid position/force control, stiffness control, the resolved acceleration based force control and the impedance control to provide an efficient tool which can be used in most of the industrial force control applications. The sensorless force control techniques are not only useful in the sense of avoiding force sensors but also in reducing the complicity of the entire control system and increasing their stability. In addition, this work present a novel sensorless force servoing techniques based on the action reaction state observer (Khalil \& Sabanovic, 2011) which allows realizing the sensorless force control without taking any measurement from the robot or the environment except a single measurement form the robot actuator. The conventional force observer requires having velocity measurement of the end-effector in contact with the environment and using it as basis for the estimation process. The action reaction state observer allows realizing the interaction force observer without measuring position or velocity of the non-collocated end-effector in contact with the environment (Khalil \& Sabanovic, n.d.), therefore allowing the force control to be realized with minimum amount of measurements from the dynamical system.

This chapter is organized as follows: Modeling of the force sensing process is included in Section 2 in order to illustrate the effect of the force sensing on the force control process. Force observers are analyzed and included in Section 3 which can be conceptually considered as alternatives for force/torque sensors. In section 4, a novel force control framework is presented which allows realization of the force control without force sensors and even with less measurements than those needed to construct the conventional force observers, thus reducing effect of the noise induced by each embedded sensor to the system. Experimental results are conducted on two experimental setups, a microsystems workstation and a single input lumped mass spring system with multiple degrees of freedom in contact with environment with varying impedance. The experimental results are distributed throughout the paper in order to provide a graphical interpretation and to support the presented ideas. Eventually, Conclusions and final remarks are included in Section 5.

\section{Modeling of force/torque sensing}

Contact between a robot end-effector with single degree of freedom and an environment in the presence of force sensing is illustrated in Fig. 1 where $m$ and $m_{s}$ are the masses of the

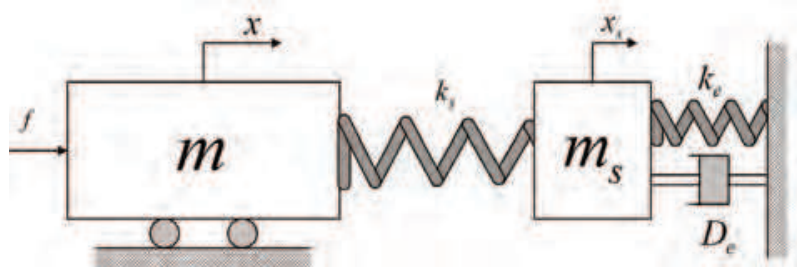

Fig. 1. Force sensor model

robot end-effector and the force sensor respectively. $k_{s}, k_{e}$ and $D_{e}$ are force sensor stiffness, environmental stiffness and the environment viscous damping coefficient, respectively. $x$ and $x_{s}$ are the positions of the robot end-effector and the force sensor, respectively. The following 
motion equations describe the contact mechanism depicted in Fig. 1

$$
\begin{gathered}
m \ddot{x}+k_{s}\left(x-x_{s}\right)+f_{\text {dist }_{1}}=f \\
m_{s} \ddot{x}_{s}-k_{s}\left(x-x_{s}\right)+f_{\text {dist }_{2}}=-f_{\text {ext }} \\
f^{\text {ext }}=z_{e} x_{s}
\end{gathered}
$$

where $z_{e}$ is the environmental impedance. $f_{\text {dist }_{1}}, f_{\text {dist }_{2}}$ and $f^{\text {ext }}$ are the disturbance forces on the first and second system degrees of freedom and the interaction force with the environment, respectively. In a regular force servoing problem the control system is designed such that the robot end-effector exerts a force $f_{\text {ext }}$ that is equal to the desired force reference $f^{\text {des }}$. It is worth noting that in the previous example the environment is assumed stationary therefore its acceleration is not included among the previous motion equations. In addition, the environmental impedance can be modeled with either an energy storage element and energy dissipation elements or both. Therefore, in the presence of a force sensor, an extra degree of freedom is added to the system. In addition, the previous model shows that the end-effector is non-collocated via an energy storage element with stiffness $k_{s}$ due to the soft structure of the force/torque sensor. The root locus of the system when the force sensor is attached to the robot end effector is depicted in Fig. 2-b, whereas, Fig. 2-a illustrates the root locus of the same system in the absence of force sensor. In both cases the root locus is plotted for different values of the environmental stiffness gain $k_{e}$. It can be easily shown from Fig. 2 that adding a force sensor to the dynamical model of the system affects the system stability dramatically. Controlling a collocated point is much easier then a non-collocated point along the dynamical system as it can be shown from Fig. 2. In order to impose the desired force on

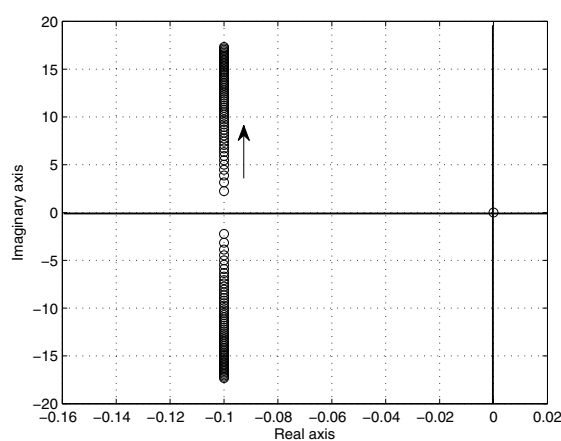

(a) collocated system

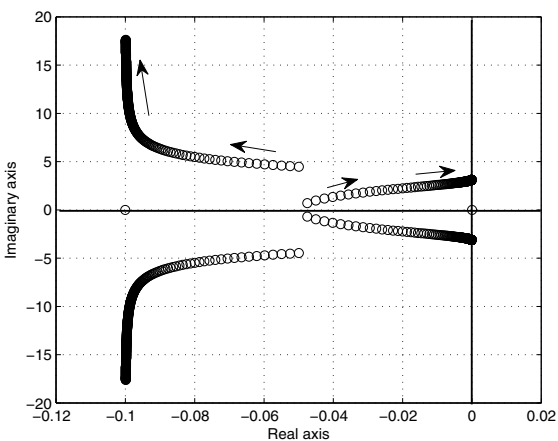

(b) non-collocated system

Fig. 2. root locus of system with and without force sensor for $k_{e}=0 \longrightarrow 300$

the environment, the force measurement taken through a force sensor has to be used to realize the motion control system as depicted in Fig. 3. However, due to the force measurement nature at which the strain is measured and amplified along with the noise $v^{s}$, the actual interaction force with the environment cannot be precisely measured. In Fig. $3, k_{v}$ and $k_{p}$ are the velocity feedback gain and proportional gains, respectively (Katsura \& Ohnishi, 2007). $k_{r}$ is the reaction force feedback gain. Due to the presence of noise in the force measurement, 
the force response of the force control system can be expressed as follows

$$
\begin{aligned}
& \frac{f_{r e f}^{\text {ext }}(s)}{f^{r e f}(s)}=\frac{k_{p} k_{f}\left(k_{e}+s D_{e}\right)}{s\left(m s+k_{v}\right)\left(m_{s} s^{2}+k_{s}+z_{e}\right)+k_{s} k_{r}\left(m s^{2}+z_{e}\right)+k_{p} k_{s}\left(k_{e}+s D_{e}\right)} \\
& \frac{f_{n}^{\text {ext }}(s)}{V^{s}(s)}=\frac{k_{p} k_{f}\left(k_{e}+s D_{e}\right)}{s\left(m s+k_{v}\right)\left(m_{s} s^{2}+k_{s}+z_{e}\right)+k_{s} k_{r}\left(m s^{2}+z_{e}\right)-k_{p} k_{s}\left(k_{e}+s D_{e}\right)}
\end{aligned}
$$

The previous transfer functions describe the force response for both the reference and force sensor noise inputs, respectively. Therefore, the total response of the system can be written as the superposition of the reference input response $f_{r e f}^{\text {ext }}(s)$ and the noise input response $f_{n}^{\text {ext }}(s)$

$$
f^{e x t}=f_{r e f}^{e x t}(s)+f_{n}^{e x t}(s)
$$

The previous equations indicate the effect of the force sensor noise on the desired force response. In addition, the force control parameters $\left(k_{v}\right)$ and $\left(k_{p}\right)$ have to be selected such that the characteristic equation of the transfer function (4) is stable. This can be easily done be using the denominator of (4) to formulate the Routh Hurwitz array and determine the gains such that stability is achieved according to the Routh Hurwitz stability criterion. The root locus of the force servoing system described by (4) is illustrated in Fig. 4 for different values of environmental stiffness $\left(k_{e}\right)$ and velocity feedback gain $\left(k_{v}\right)$. In general, environmental stiffness and the velocity feedback gain are of great importance for any force control problem since the cause of instability is that the environmental stiffness matrix increases the equivalent position gain of the control system. Therefore, the velocity gain has to be chosen not only based on the position gain but also on the large environmental stiffness. Otherwise the resulting system will be highly under damped and possibly unstable (Karunakar \& Goldenberg, 1988). The previous analysis indicates how the utilization of

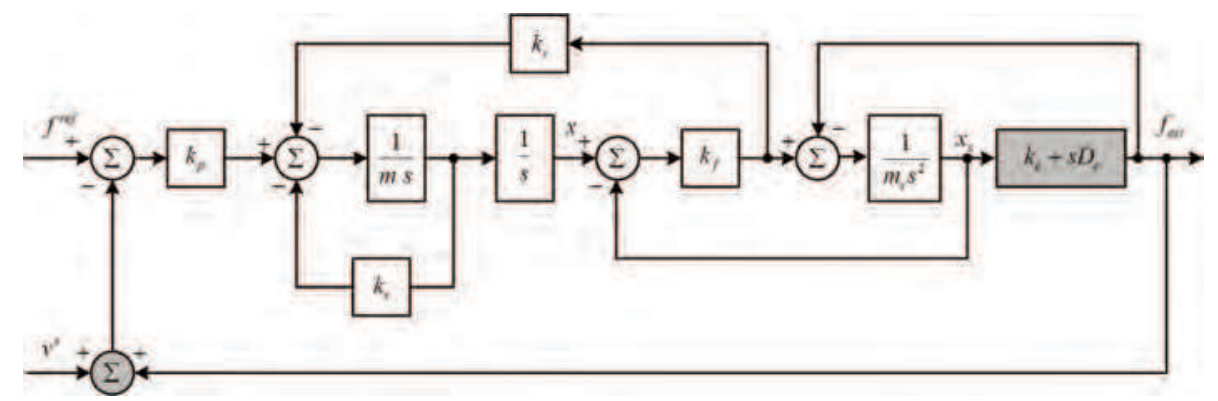

Fig. 3. Robot in contact with environment with force sensing

force sensors adds an extra degree of freedom to the control system, this in turn makes the robot end-effector non-collocated from its actuator. In addition, the response of the desired interaction force is affected by the noise input given by (5). Therefore, modeling of the force sensing shows how problematic the force sensing is, due to the non-collocation, the extra degree of freedom and the measurement noise. Before going into how to provide the sensorless force control techniques, we have to consider a more general form of the problem, 


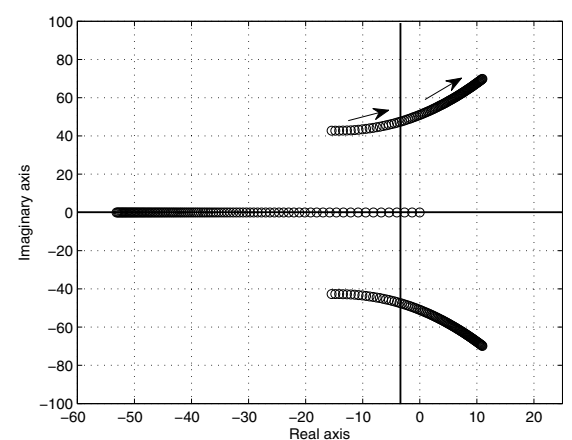

(a) $k_{r}=1 \longrightarrow 10000$

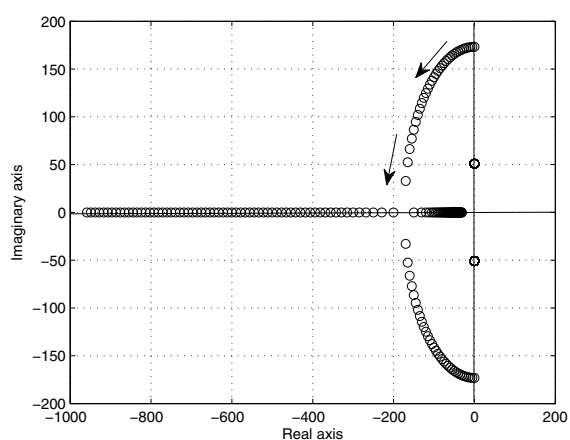

(b) $k_{v}=1 \longrightarrow 2000$

Fig. 4. root locus of system with force servoing

the generalized equation of motion of a robot manipulator can be therefore written as,

$$
\begin{gathered}
\mathrm{M}(q) \ddot{q}+\mathrm{V} \dot{q}+\mathrm{C}(q, \dot{q})+\mathrm{G}(q)+\mathrm{J}^{t} f^{e x t}=\tau \\
m_{s} \ddot{x}_{s}+k_{s}\left(x_{e}-x_{s}\right)-z_{e} x_{s}=0
\end{gathered}
$$

where, $q, \dot{q}$ and $\ddot{q}$ are the joint position, velocity and acceleration vectors respectively. $\tau$ is the generalized joint torque vector. $\mathrm{M}(q)$ is the inertia matrix. V is the viscous damping matrix, $C(q, \dot{q})$ and $G(q)$ are the centrifugal and coriolis forces and gravity vectors, respectively. $\mathrm{J}$ is the robot Jacobian and $f^{e x t}$ is the interaction force with the environment. $x_{e}$ is the robot end effector position. It will be shown in the upcoming sections that force observers and the other sensorless force control techniques will allow simplifying the dynamical model as the extra degree of freedom can be avoided.

\section{Force/torque observers}

In order to utilize force observers instead of the force sensors, we will first start with analyzing the disturbance observer structure since the reaction force observer is depending on the disturbance observer techniques. The conventional structure of the disturbance observer is depicted in Fig. 5. As depicted in Fig. 5, the reference input $(u)$ along with the output $(y)$ of the actuator can be used to estimate the incident disturbances $(d)$. $G(s)$ represents the model of the actuator while $\left(G_{n}(s)\right)$ represents the nominal model of the actuator. Therefore, disturbance can be computed as follows with force or torque units

$$
\begin{aligned}
d_{o}(s) & =u(s)-\mathrm{G}_{n}^{-1}(s)(y(s)-v(s)) \\
& =\left(\mathrm{G}^{-1}(s)-\mathrm{G}_{n}^{-1}(s)\right) y(s)+d(s)+\mathrm{G}_{n}^{-1}(s) v(s) \\
& =\Delta \mathrm{G}(s) y(s)+d(s)+\mathrm{G}_{n}^{-1}(s) v(s)
\end{aligned}
$$

where, $\Delta G(s)$ is the real plant perturbation from the nominal one. Due to the presence of derivatives in the inverse plant dynamics $\left(\mathrm{G}^{-1}(s)\right)$, the disturbance force has to be estimated 


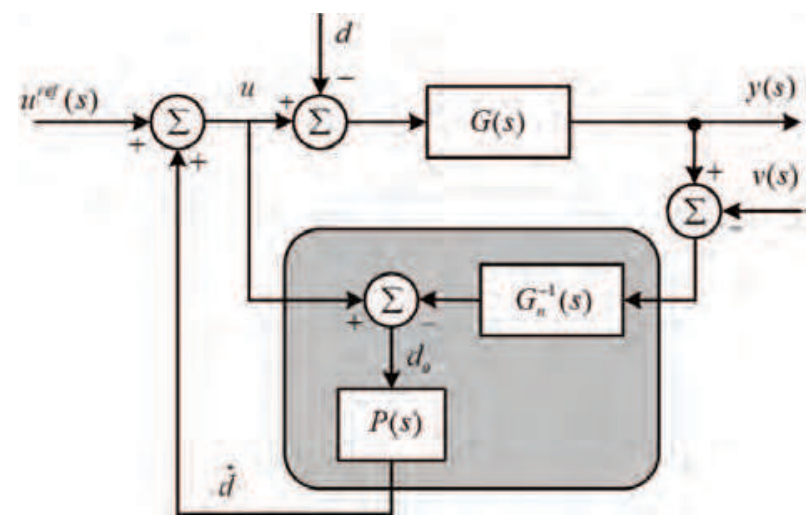

Fig. 5. Disturbance observer

through a low-pass filer as follows

$$
\widehat{d}(s)=\mathrm{P}(s) d_{o}(s)
$$

where $(\mathrm{P}(s))$ is the low-pass filter associated with the disturbance observer. In general, disturbance can be modeled by

$$
\frac{d^{k} d_{o}(t)}{d t^{k}}=0
$$

and upon the degree of the polynomial used to model the disturbance signal, the order of the low-pass filter is determined. $k$ is an integer that indicates the model of the disturbance (step, ramp, parabolic, etc) and can be further used in the selection of $\mathrm{P}(s)$. This in turn determines the order of the disturbance observer. Regardless to the order of the disturbance observer and the type of the disturbance signal, (9) along with (10) indicate that the estimated disturbance signal consists of the incident reaction disturbance force $d(s)$ along with parameter deviation induced disturbances $\Delta \mathrm{G}(s) y(s)$ and disturbance due to the sensor noise $G_{n}^{-1}(s) v(s)$. Therefore, when the robot's end effector comes into contact with an environment, the interaction force is coupled in the disturbance signal. Therefore, reaction force observer depends on the disturbance observer structure expect that the other terms of the disturbance have to be subtracted in order to decouple the interaction forces from the disturbance signal. For instance, disturbance observer can be applied on (7) in order to estimate the disturbances, in this case disturbance force includes a coupled information which consists of the viscous damping friction, centrifugal and coriolis forces friction forces, gravity forces and external forces along with the parameter deviation induced disturbances, namely, the self-varied inertia and the torque ripple. Therefore, reaction force observer requires performing parameter identification in order to decouple the reaction force from the disturbance signal. According to (7), disturbance can be expressed as follows

$$
d=\mathrm{V} \dot{q}+C(q, \dot{q})+G(q)+\mathrm{J}^{t} f^{e x t}+\Delta \mathrm{M}(q) \ddot{q}-\Delta k_{t} I_{a}^{r e f}
$$

where $\Delta \mathrm{M}(q) \ddot{q}$ and $\Delta k_{t} I_{a}^{r e f}$ are the actuator self-varied inertia and torque ripple. Disturbance observer is generally implemented on the joint space of the robot at which an actuator is 
located. Therefore, the last two terms of the disturbance force are related to the actuator parameters. $k_{t}$ and $I_{a}^{r e f}$ are the actuator torque constant and reference current respectively. It is worth noting that equation (12) can be implemented on each joint of the robot. As shown in Fig. 5, the estimated disturbance with force or torque units is commonly used in the realization of an additional compensation control input which is used in the attainment of robustness.

In order to decouple the reaction force out of the disturbance force, or in order to construct a force observer for the realization of the sensorless force control, system parameters have to be identified. The constant angular velocity motion test (Murakami et al., 1993) can be used in order to estimate the gravity and friction forces at each degree of freedom of the system. A parameter identification process was proposed in (Khalil \& Sabanovic, 2010a), in order to observe the self-varied inertia and the torque ripple online (Ohnishi et al., 1996). These parameter identification procedures have to be performed and used to assist in constructing the force observer. The estimated reaction force can be expressed as,

$$
\widehat{f}^{e x t}=\frac{g_{\text {reac }}}{s+g_{\text {reac }}}\left(I_{a}^{r e f} k_{t n}+g_{\text {reac }} m_{n} \dot{q}-\mathrm{V} \dot{q}-C(q, \dot{q})-G(q)\right)-g_{\text {reac }} m_{n} \dot{q}
$$

$m_{n}$ and $k_{t n}$ are the nominal actuator inertia/mass and torque/force constant. The interaction force with the environment is observed through a low-pass filter with a cut-off frequency $g_{\text {reac }}$. It can be easily concluded from (13) that the force observer depends on a single measurement from the actuator along with the reference current input. Therefore, the problems associated with force sensors can be avoided by utilization of the force observer (13). There exist two main points that we have to emphasize here about force observers, namely, the force observer sensitivity and the tradeoff between the response and the noise attenuation. Concerning with the second point, it can be easily shown that the reaction force is estimated through a low-pass filter in order to assist in attenuating the effect of noise amplification due to differentiation. Therefore, the bandwidth of the force observer or even the disturbance observer is limited with the bandwidth of the sensor noise. On the other hand, sensitivity of the reaction force observer is related to the accuracy of the position or velocity measurement. For instance, Fig. 6 indicates the force observer sensitivity for micro scale interaction forces. The observed force peak shown in Fig. 6 was obtained experimentally through a force observer of the form (13) based on position sensor with 10nm accuracy. This indicates that sensitivity of the disturbance or reaction force observer depends on the precision of the velocity or position measurement. The Block diagram representation of the force servoing control system with force observer is depicted in Fig. 7. Force sensor is altered with the force observer, therefore the noise associated with the force sensor, the non-collocation and the complicity of the associated electronic setups and wirings are all avoided. Although the bandwidth of the control system can be relatively increased when force observer is utilized, still the noise added due to the the velocity measurement defines the new bandwidth of the force observer based force servoing system. In the experimental result illustrated in Fig. 6, the force observer requires the velocity information which was obtained by differentiating the position encoder signal through a low-pass filter with cut-off frequency $g_{l}$ to attenuate the effect of noise amplifications due to differentiation, experimental values of the force observer gain and the low-pass filter gains are included in table. 1.

So Far, the previous analysis indicates that it is possible to alter force sensing with force observer. However, the main drawback in this procedure is that the bandwidth of the force observer based force servoing control system depends on the bandwidth of the noise 


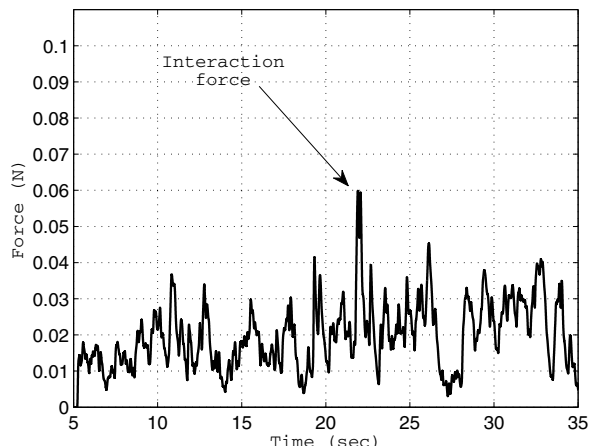

(a) interaction force

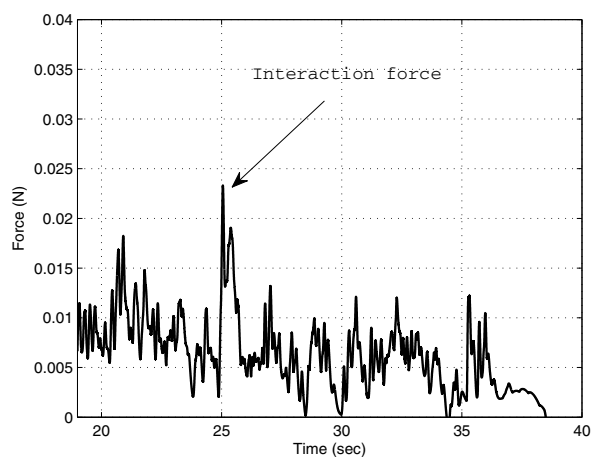

(b) interaction force

Fig. 6. Sensitivity of reaction force observer for micro scale interaction force

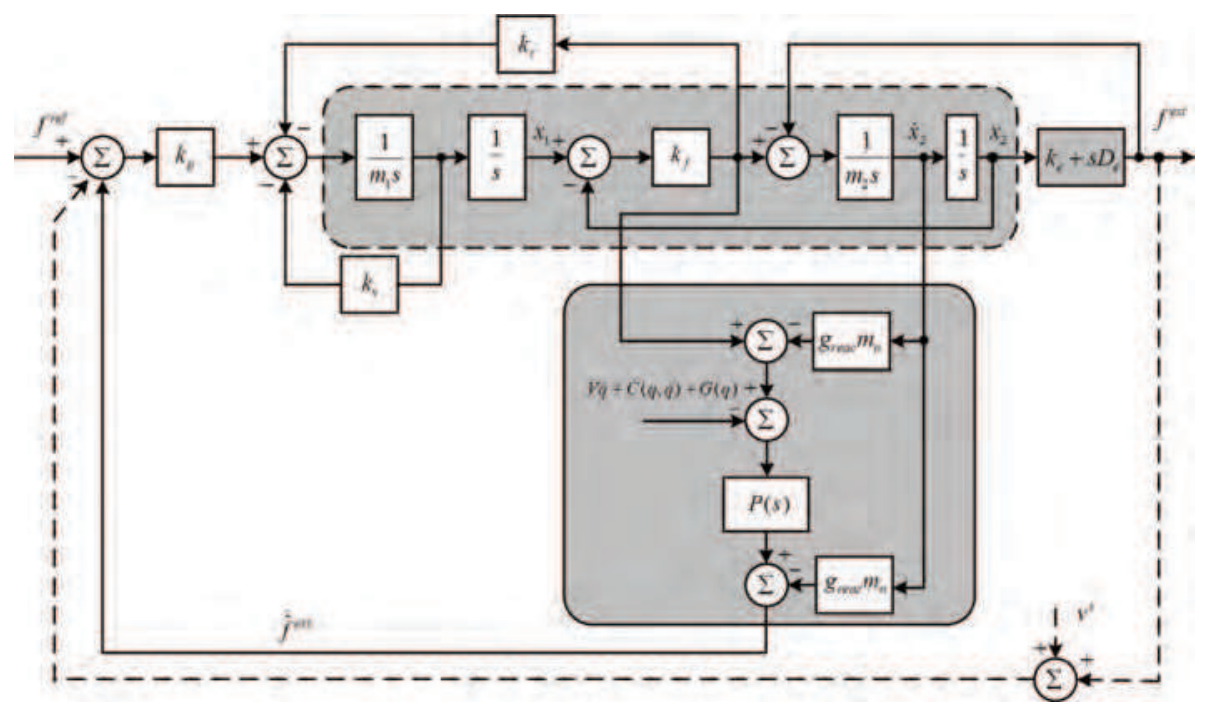

Fig. 7. Force servoing using reaction force observer

associated with the measured velocity signal. Although the force observer spares the control system from having a force measurement, it necessitates having a velocity measurement as shown in Fig. 7. This means that the force observer alters the force observer with a position or velocity sensor. Indeed, the noise associated with the position or velocity sensors is less than the noise associated with force sensor. Nevertheless, one would like to spare the control system having position or velocity sensor attached to the robot end-effector. With the observer presented in (Khalil \& Sabanovic, 2010b;b), the position and velocity of the non-collocated end-effector can be observed from measurements taken from the input (actuator) of the dynamical system. This in turn, spares the non-collocated end-effector of the robot having any attached sensors. 
In the next Section, a novel observer is introduced based on the action reaction law of dynamics in order to allow realization of the force observer without taking the velocity measurement from the dynamical system but rather its estimate.

\section{Action reaction force control}

It was shown in the previous Section that the force control can be realized without force sensor utilization through force observers. Therefore, the word sensorless force control does mean that force sensors is not utilized but it does not mean that sensors are not utilized since one has to measure or sense some signal from the system and use it as basis for the estimation process. The force observer explained in the previous section depends on measurement taken from the dynamical system which interacts with the environment, i.e., the velocity of the subsystem in contact with the environment. In this section, we will further try to avoid taking this measurement $\left(\dot{x}_{2}\right)$ and /or $\left(x_{2}\right)$.

It was shown in (O'Connor, 2003; 2007b), that sensorless control of flexible systems can be realized if the propagating mechanical waves throughout any dynamical system were considered as natural feedback (O'Connor, 1998; 2007a). This wave based approach depends on launching and absorbing mechanical waves with the right amount at the right time in order to position a non-collocated end-effector to a target position without residual vibration. However, the wave based approach requires having an additional measurement from a non-collocated mass along the flexible lumped system in order to determine the propagating mechanical waves along the system, in this section we would like to find a method to avoid this measurement. In (Khalil \& Sabanovic, 2010b) the action reaction well-known law of dynamics was utilized in order to provide an instantaneous feedback-like force from a class of dynamical system with inaccessible outputs. The proposed action reaction state observer in (Khalil \& Sabanovic, 2010b) allows realization of the previous force observer without measuring $\dot{x}_{2}$ or $x_{2}$. The action reaction state observer can be implemented for a class of dynamical system of the following form

$$
\begin{aligned}
& \dot{\mathrm{x}}=\mathrm{Ax}+\mathrm{B} u \\
& \mathrm{y}=\mathrm{Cx}+\mathrm{D} u
\end{aligned}
$$

which can be shown to have the following structure

$$
\begin{gathered}
\dot{\mathrm{x}}_{a}=\mathrm{A}_{a} \mathrm{x}_{a}+\mathrm{B}_{a} u_{a}+\mathrm{B}_{\text {reac }} f_{\text {reac }}\left(\mathrm{x}_{\mathrm{a}}, \dot{\mathrm{x}}_{a}, \mathrm{x}_{\mathrm{p}}^{1}, \dot{\mathrm{x}}_{p}^{1}\right) \\
\dot{\mathrm{x}}_{p}=\mathrm{A}_{p} \mathrm{x}_{p}+\mathrm{B}_{p} f_{\text {reac }}\left(\mathrm{x}_{\mathrm{a}}, \dot{\mathrm{x}}_{a}, \mathrm{x}_{p}^{1}, \dot{\mathrm{x}}_{p}^{1}\right)
\end{gathered}
$$

where $\mathrm{x} \in \mathrm{R}^{n}$ and $\mathrm{y} \in \mathrm{R}^{m}$ are the state and measurement vectors, respectively. $\mathrm{A}, \mathrm{B}, \mathrm{C}$ and $\mathrm{D}$ are the system matrix, distribution vector of input, observation column vector and feed forward matrix with the appropriate dimensions, respectively. The dynamical system we consider consists of two subsystems, the first subsystem is the single input while the second is the flexible subsystem with the subscripts $(a)$ and $(p)$, respectively. $x_{a}$ and $x_{p}$ are the actuator and plant state vectors, respectively. Actuator states can be measured, whereas, the flexible dynamical subsystem states are inaccessible. $f_{\text {reac }}\left(\mathrm{x}_{\mathrm{a}}, \dot{\mathrm{x}}_{a}, \mathrm{x}_{\mathrm{p}}^{1}, \dot{\mathrm{x}}_{p}^{1}\right)$ is the incident instantaneous reaction force on the actuator. $x_{p}^{1}$ and $\dot{x}_{p}^{1}$ are the position and velocity of the first non-collocated degree of freedom along the flexible subsystem. $\mathrm{B}_{\text {reac }}$ is the reaction force distribution vector. $\mathrm{B}_{a}$ is the actuator input distribution vector and $\mathrm{B}_{p}$ is the plant reaction 
force distribution vector. The action reaction state observer consists of the following three observers

$$
\begin{gathered}
\widehat{d}=\frac{g_{\text {dist }}}{s+g_{\text {dist }}}\left[g_{\text {dist }} m_{a n} \dot{\mathrm{x}}_{a}+i_{a} k_{f n}\right]-g_{\text {dist }} m_{a n} \dot{\mathrm{x}}_{a}=\frac{g_{\text {dist }}}{s+g_{\text {dist }}}\left[i_{a} k_{f n}-s m_{a n} \dot{\mathrm{x}}_{a}\right]=\frac{g_{\text {dist }}}{s+g_{\text {dist }}} d(17) \\
\widehat{f_{\text {reac }}}\left(i_{a}, \dot{\mathrm{x}}_{a}\right)=\frac{g_{\text {reac }}}{s+g_{\text {reac }}}\left[g_{\text {reac }} \widehat{\Delta m_{a}} \dot{\mathrm{x}}_{a}+i_{a} \widehat{\Delta k_{f}}+\widehat{d}\right]-g_{\text {reac }} \widehat{\Delta m_{a}} \dot{\mathrm{x}}_{a} \\
\dot{\mathrm{x}}=\mathrm{A} \widehat{\mathrm{x}}+\mathrm{B} u+\mathrm{M}\left(\widehat{f_{\text {reac }}}\left(\mathrm{x}_{\mathrm{a}}, \dot{\mathrm{x}}_{a}, \mathrm{x}_{\mathrm{p}}^{1}, \dot{\mathrm{x}}_{p}^{1}\right)-f_{\text {reac }}\left(\mathrm{x}_{\mathrm{a}}, \dot{\mathrm{x}}_{a}, \widehat{\mathrm{x}}_{\mathrm{p}}^{1}, \dot{\mathrm{x}}_{p}^{1}\right)\right)
\end{gathered}
$$

Structure of the action reaction state observer is depicted in Fig. 8, this observer can be utilized in order to estimate the dynamical states of a subsystem at which measurements cannot be made. $g_{\text {dist }} \in \mathrm{R}^{+}, g_{\text {reac }} \in \mathrm{R}^{+}$and $\mathrm{M} \in \mathrm{R}^{n \times 1}$ are the action reaction state observer scaler

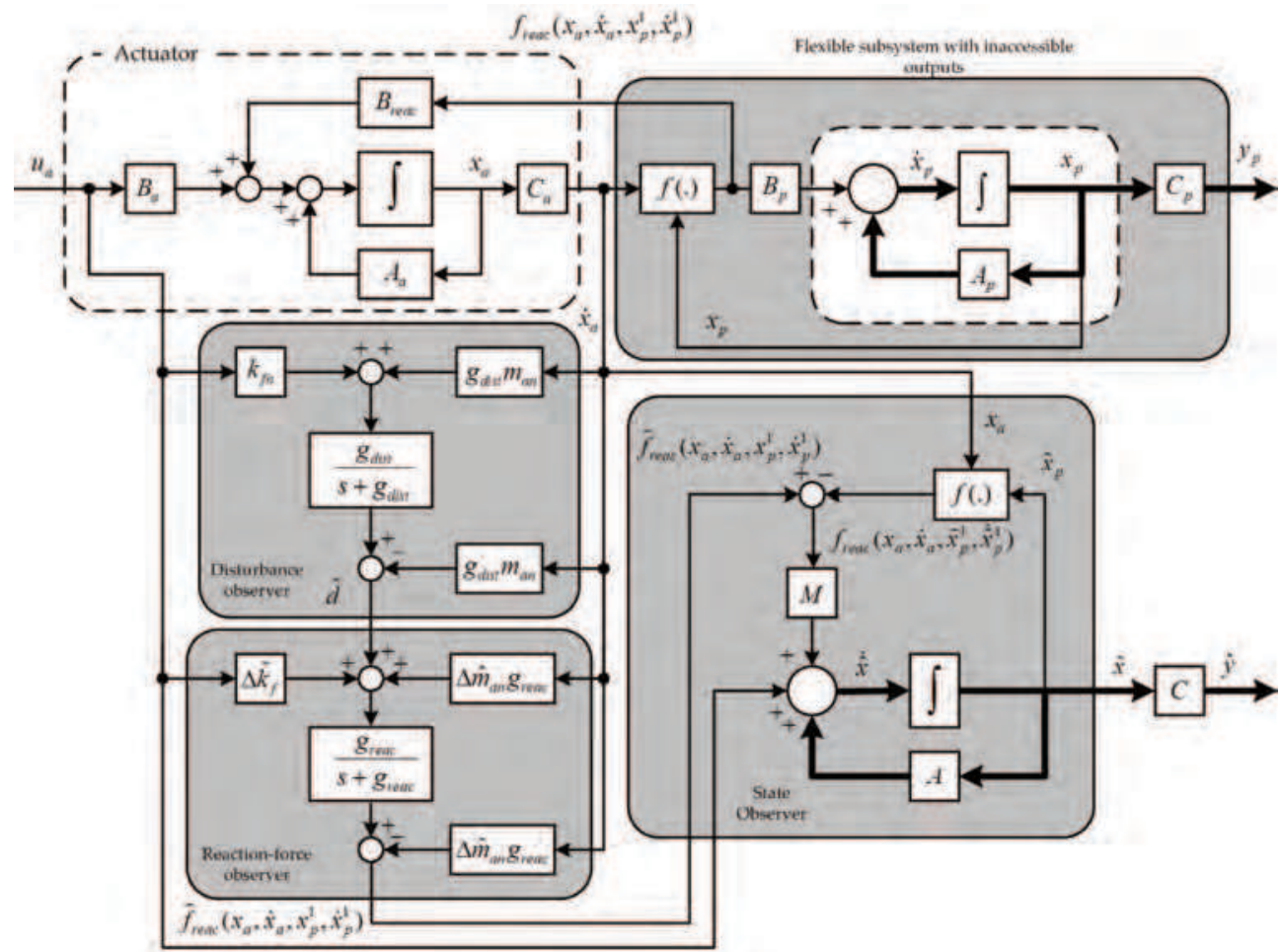

Fig. 8. Action reaction state observer

and vector gains respectively. The design of the action reaction state observer is not trivial since it depends on the function $(f()$.$) which can be determined upon the dynamics of the$ interacting systems, e.g., if the systems denoted with the subscript (a) and (b) are connected by the mean of a linear elastic element with stiffness $k$, then the function $f($.$) can be expressed$ as $f()=.k\left(x_{a}-x_{p}\right)$, here $\left(x_{a}-x_{p}\right)$ represents the deflection in the elastic element due to the interaction of the two systems. If the two previous systems are connected via an elastic element and energy dissipation element with linear viscous damping $c$, then the function 
$f($.$) can expressed as f()=.k\left(x_{a}-x_{p}\right)+c\left(\dot{x}_{a}-\dot{x}_{p}\right)$. if the coupling between the previous systems is defined by a non-linear spring with large deflection $(y)$, the incident reaction force can be defined as $f()=.k\left(1-a^{2} y^{2}\right) y$. Therefore, upon the function $f($.$) , error dynamics$ of the action reaction state observer can be determined. Obviously, the action reaction state observer gains $g_{\text {dist }}, g_{\text {reac }}$ and $\mathrm{M}$ have to be selected such that the error dynamics of the observer is stable. It can be shown from Fig. 8 that the action reaction state observer consists of three observers in cascade, namely, disturbance force observer, reaction force observer and a Luenberger-like state observer. It is worth noting that the action reaction state observer shown in Fig. 8 is based on first order disturbance observer, first order reaction force observer and $n-2$ order Luenberger-like state observer, where $n$ is the order of the dynamical system (14). The order of the disturbance and reaction force observers depend on the order of the polynomial used to model the disturbance force (11). Therefore, different structures of the action reaction state observer can be designed upon the nature of the disturbance force. Fig. 9 illustrates experimental results of state estimation using the action reaction state observer. This experiment is conducted on a dynamical subsystem (16) with 2 degrees of freedom driven by a single input described by (15). Position and velocity of each degree of freedom of (16) are

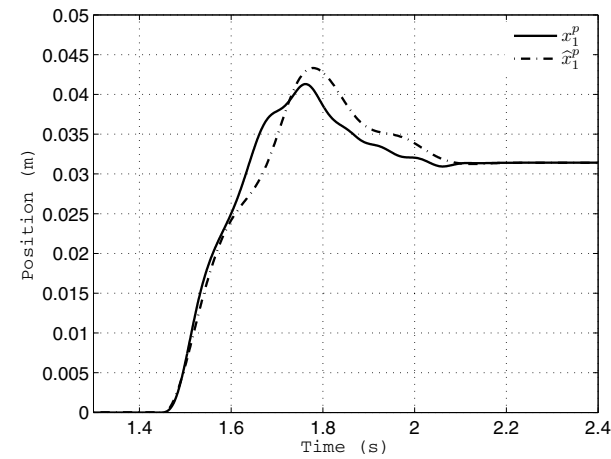

(a)

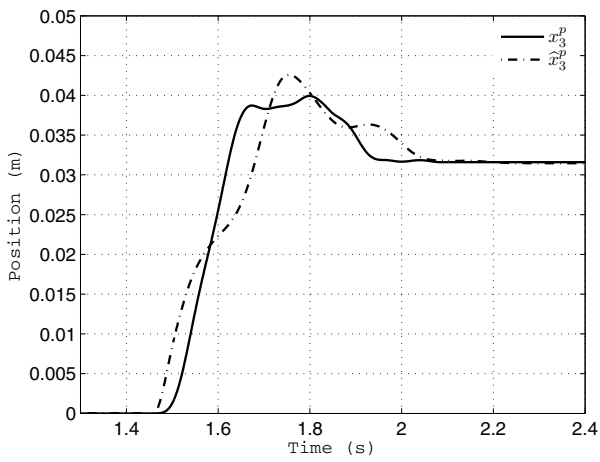

(c)

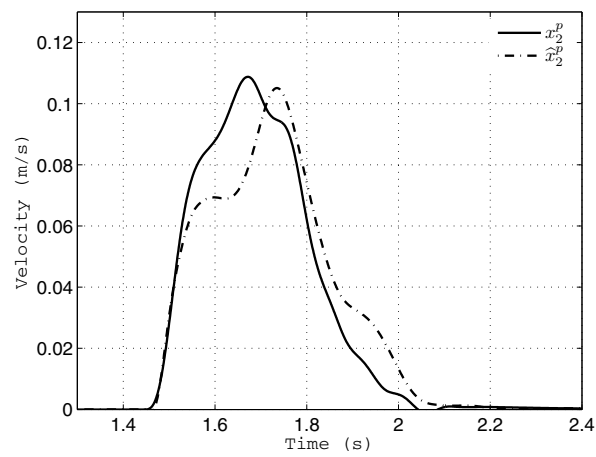

(b)

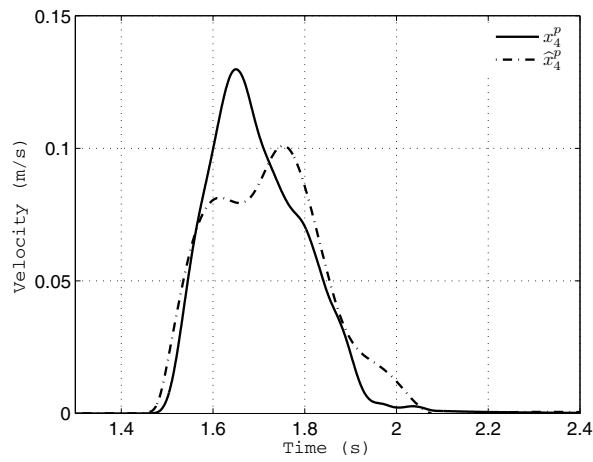

(d)

Fig. 9. State estimation experimental result using the action reaction state observer 
estimated using the action reaction state observer as shown in Fig. 9 where the estimated states are plotted versus the measured ones in order to verify the validity of the action reaction state observer. The previous experimental result depicted in Fig. 9 indicates that the measurements $\left(\dot{x}_{2}\right)$ and $\left(x_{2}\right)$ can be replaced by their estimates obtained through the action reaction state observer. Therefore, the reaction force observer depicted in Fig. 7 can be further modified by incorporating the action reaction state observer in order to provide estimates of the states $\left(\dot{x}_{2}\right)$ and $\left(x_{2}\right)$. The action reaction state observer is utilized along with the force observer with cut-off frequency $\left(g_{f o}\right)$ as shown in Fig. 10 in order not to measure $\left(\dot{x}_{2}\right)$ and $\left(x_{2}\right)$, their estimates $\left(\dot{\hat{x}_{2}}\right)$ and $\left(\widehat{x}_{2}\right)$ obtained through the action reaction state observer are used instead. It can be

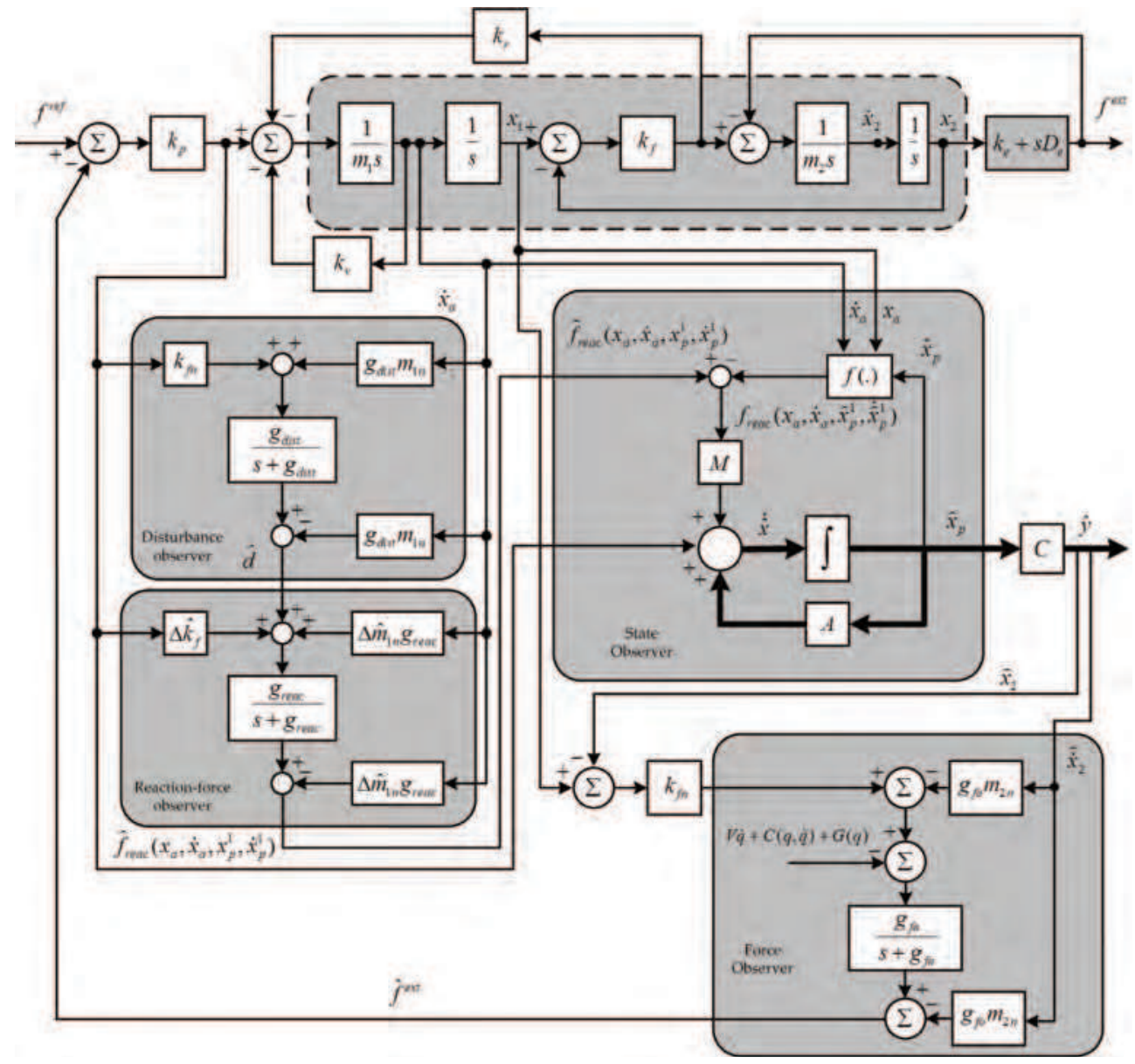

Fig. 10. Action reaction based force control

easily shown from Fig. 10 that the sensorless force control can be realized not only in the absence of the force measurement but also in the absence of the velocity and position of the non-collocated mass in contact with the environment. Therefore, the previous action reaction based force observer avoids the noise associated with force sensing and avoids the noise 
associated with measuring the end effector (might be non-collocated) position or velocity. The dynamical system depicted in Fig. 10 can be considered as a two mass resonant system in contact with an environment with impedance $z_{e}$ and the action reaction based force servoing control system shown in the same figure can be considered as a sensorless control system since measurements required to realize the force control are reduced to a single measurement taken from the first degree of freedom, generally this degree of freedom stands for the actuator, i.e., force control can be realized from measurements taken from the actuator whereas the reset of the dynamical system in contact with the environment is kept free from any measurements. Strictly speaking, there exist a measurement taken from the dynamical system in contact with the environment rather than the actuator measurements, namely, the incident reaction forces $\left(f_{\text {reac }}\left(\mathrm{x}_{\mathrm{a}}, \dot{\mathrm{x}}_{a}, \mathrm{x}_{\mathrm{p}}^{1}, \dot{\mathrm{x}}_{\mathrm{p}}^{1}\right)\right)$ which can be estimated as shown previously using a disturbance observer and a reaction force observer in cascade. The central idea of the action reaction based force servoing is based on the well-known action reaction principle of dynamics. The dynamical system we considered in the previous analysis is a two mass resonant system, the second degree of freedom is in contact with an environment. Therefore, whenever the second degree of freedoms (end-effector) comes into contact with the environment the instantaneous reaction can be observed if the velocity of the second degree of freedom is measured as shown in Fig. 7 . In order to keep the second degree of freedom or the end effector even free from this velocity or position measurement, the action reaction state observer is utilized to provide the force observer with the velocity and/or the position estimates of the second degree of freedom, thus realizing the force control from measurement taken from the active degree of freedom of the system, namely the actuator.

Application of the sensorless force control techniques and the action reaction based force servoing are oriented toward systems at which measurements cannot be made such as microsystems and micromanipulation operations. In these specific application, sensor utilization is commonly believed to be one of the major reasons for not having these systems automated till now as it was reported in (Savia \& Koivo, 2009). A micromanipulation system is shown in Fig. 11 which consists of a master side and a remote slave side used for biological cell characterization. The end effector of the slave robot is used in manipulating, injecting and characterization of biological cells through scaled bilateral teleoperations. Such applications requires measuring the interaction forces in order to achieve safe interaction with these fragile cells or any similar objects. However, the force sensing is very problematic due to the reasons listed and discussed in the previous sections. In addition, due to the very limited workspace, having force sensor embedded to the end effector of the remote slave robot is hard to be achieved. The current ongoing research in the area of microsystems and micromanipulation is based on utilization of the AFM probes which are expensive and have the same typical problem listed in the previous sections. Many authors on the other hand tried to utilize visual feedback in order to realize the interaction forces between the end-effector and biological cells, however this process cannot provide online estimated force feedback which is needed to realize the force control (Huang et al., 2007). Therefore, the sensorless force control techniques proposed in this section can be utilized in such application in order to spare the remote slave robot from having force sensors attached to it. In addition, utilization of these sensorless force control techniques reduces the complicity of the entire control system.

In the realization of the force control, the estimated forces are used instead of the measured forces. However, a verification is always required in order to demonstrate that the estimated forces are converging to the actual ones. This can be done by comparing the actual forces 


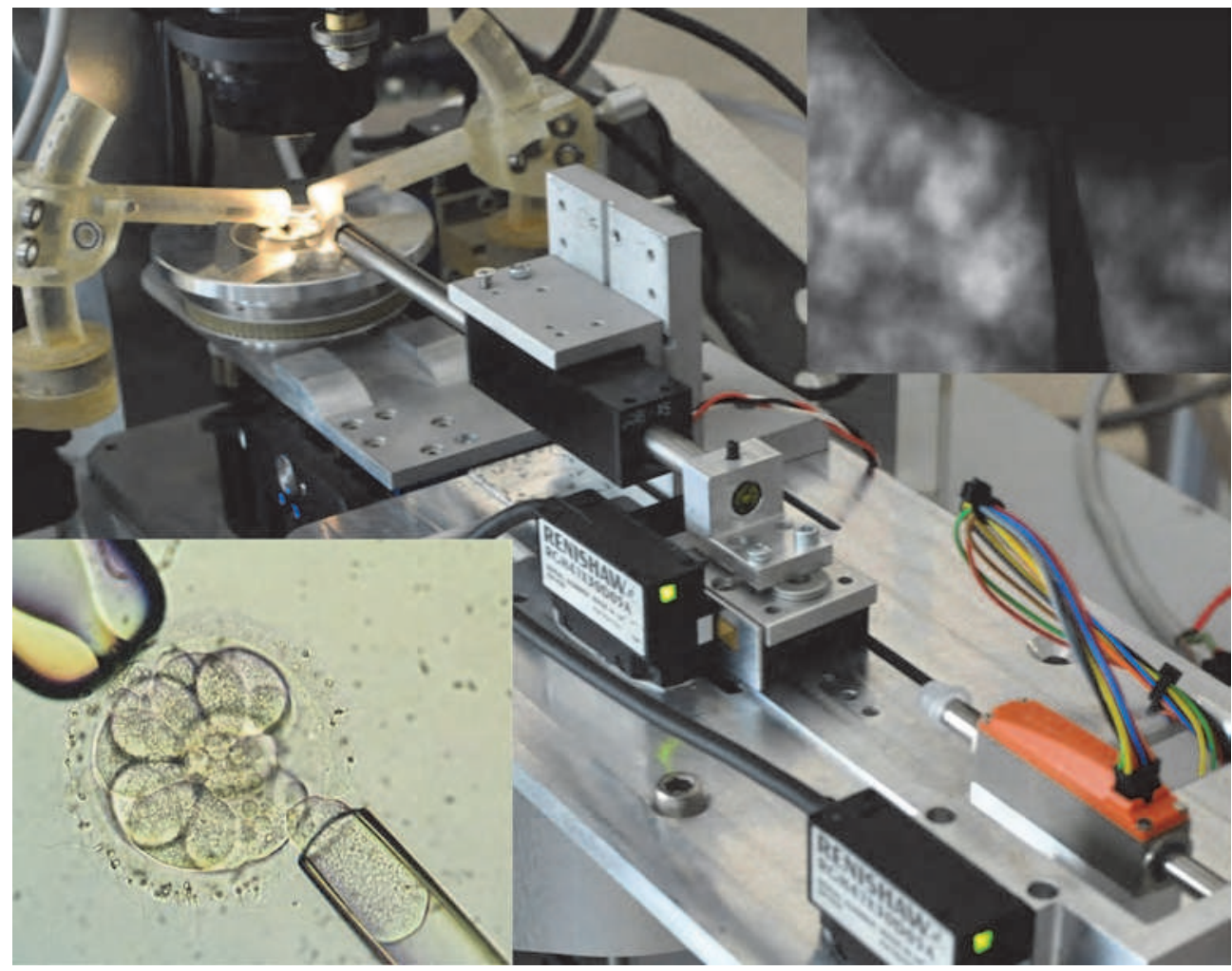

Fig. 11. Micro-system experimental setup for sensorless force control operations on biological cells

with the estimated ones. Similar to the experimental result conducted to verify the validity of the action reaction state observer, another experiment has to be performed in order to compare the estimated forces obtained through the action reaction force observer depicted in Fig. 10. Experimentally, a lumped mass spring system with three degrees of freedom is used in order to verify the validity of the action reaction based force observer. The lumped mass spring system has a single input from which measurements can be taken, namely the velocity of the actuator along with the current reference input. The single input is labeled with the symbol (A) as depicted in Fig. 12, at the end effector of the lumped system another actuator (B) is mounted and conceptually considered as an environment with impedance $\left(z_{e}\right)$. Thus using actuator or the environment (B) the impedance can be varied and experiments can be conducted easily on a soft and hard environments by changing the impedance of actuator (B). The sensorless force control can be achieved by forcing the end effector of the lumped mass spring system (The last non-collocated mass) to exert the desired force on the environment (B) without using force sensors, the force servoing sensorless control framework depicted in Fig. 7 requires measuring the velocity of the end effector and using it as basis of the force observer, whereas, the action reaction based force servoing approach depicted in Fig. 10 does not require taking this measurement or any measurement from the dynamical 
subsystem attached to the single input (A). This means that the sensorless force control is realized from measurements taken from actuator (A), whereas the dynamical subsystem is kept free from any measurement and attached sensors. The parameters of this experiment are included in Table. 1. Optical encoders with $1 \mu \mathrm{m}$ resolution are attached to each degree of freedom as depicted in Fig. 12 in order to compare the estimated states with the actual ones. These encoder signal are just used for the sake of verification only.

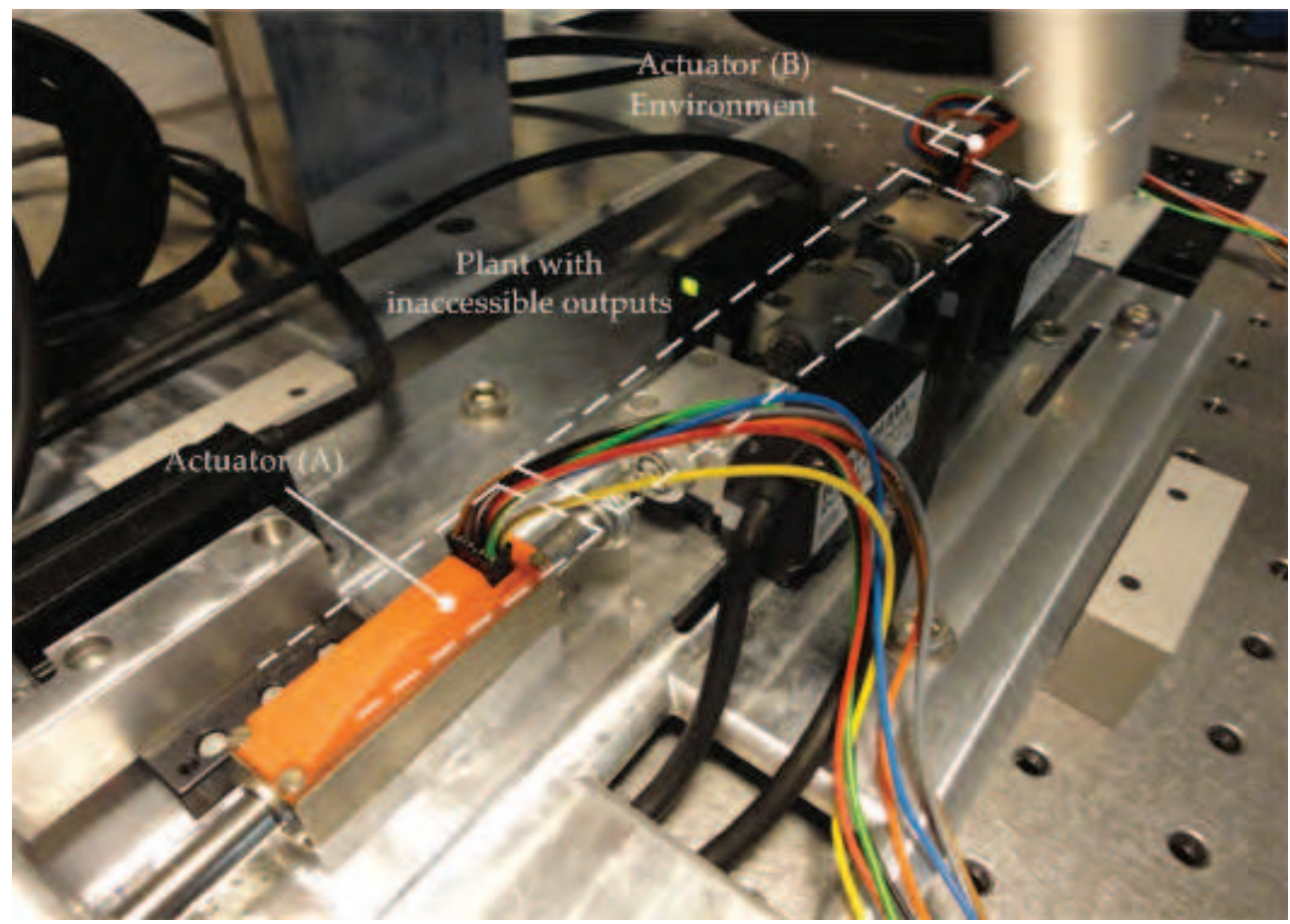

Fig. 12. Sensorless force control experimental setup

The experimental results of the action reaction external force estimation are depicted in Fig. 13 and Fig. 14 at which the estimated interaction force using the action reaction based force observer is compared with the measured interaction forces. The experiments were performed in different frequencies and amplitudes and in each case the actual interaction force was compared with the estimated one in order to verify the validity of the proposed action reaction based force observer. The experimental results shown in Fig. 13 and Fig. 14 indicate that the estimated interaction force $\left(\hat{f}^{e x t}\right)$ can further be used in the realization of the sensorless force control as depicted in Fig. 10. Fig. 14 illustrates the force estimation experimental result at higher frequency of the external interaction force, this result shows that the observer satisfactory operate in a wide frequency range of the dynamical system.

The force observer depicted in Fig. 10 consists of four observers in cascade, namely the disturbance force, reaction force, the Luenberger-like state observer and the force observers. Therefore, the action reaction based force observer has four degrees of freedom or scaler and vector gains which have to be selected properly to accomplish stable convergence of the 


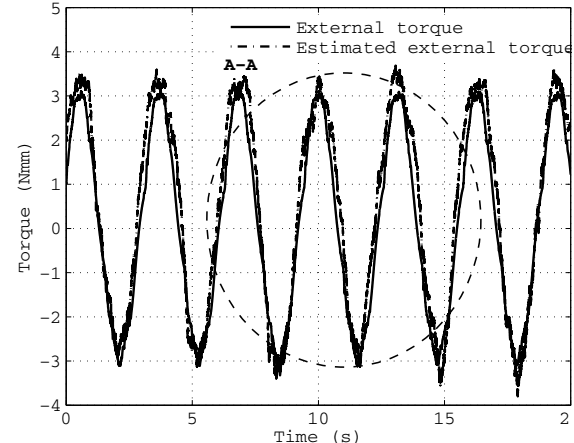

(a)

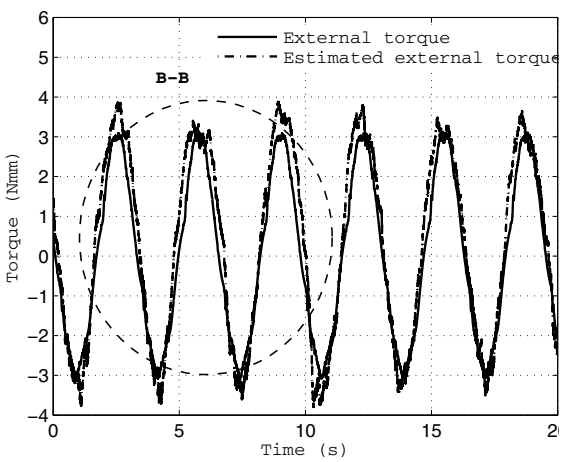

(c)

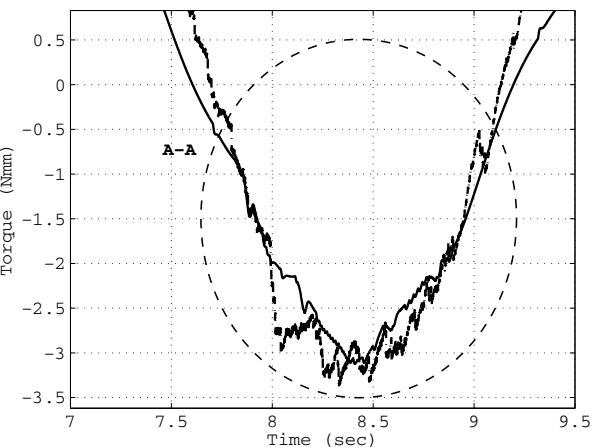

(b)

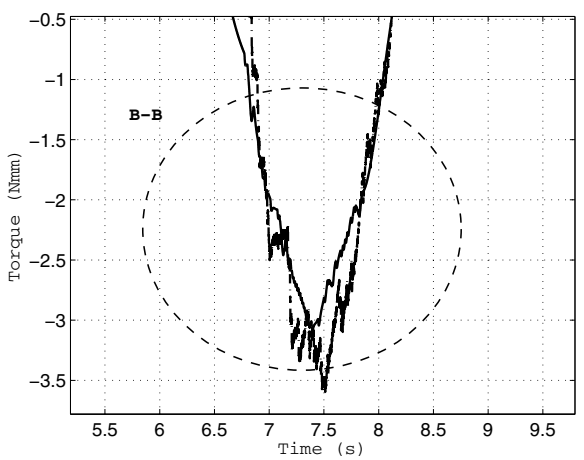

(d)

Fig. 13. Action reaction based force estimation experimental results

\begin{tabular}{|c||c|c|c|}
\hline Actuator force constant & $k_{f n}$ & 6.43 & $\mathrm{~N} / \mathrm{A}$ \\
\hline Actuator Nominal mass & $m_{a n}$ & 0.059 & $\mathrm{~kg}$ \\
\hline Lumped masses & $m_{1,2,3}$ & 0.019 & $\mathrm{~kg}$ \\
\hline Identified spring constants & $k_{1,2,3}$ & 503.96 & $\mathrm{~N} / \mathrm{m}$ \\
\hline Identified viscous damping coefficients & $c_{1,2,3}$ & 0.262 & $\mathrm{Ns} / \mathrm{m}$ \\
\hline Reaction force observer gain & $g_{\text {reac }}$ & 628 & $\mathrm{rad} / \mathrm{s}$ \\
\hline Disturbance observer gain & $g_{\text {dist }}$ & 628 & $\mathrm{rad} / \mathrm{s}$ \\
\hline Force observer gain & $g_{f o}$ & 628 & $\mathrm{rad} / \mathrm{s}$ \\
\hline \hline Low-pass filter gain & $g_{l}$ & 1000 & $\mathrm{rad} / \mathrm{s}$ \\
\hline Sampling time & $T_{s}$ & 1 & $\mathrm{~ms}$ \\
\hline
\end{tabular}

Table 1. Experimental and simulation parameters 


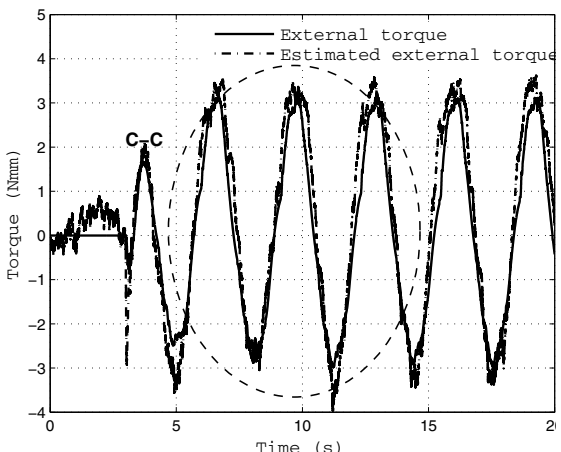

(a)

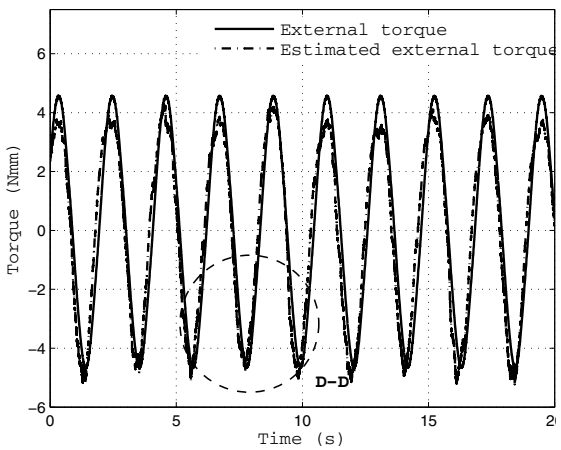

(c)

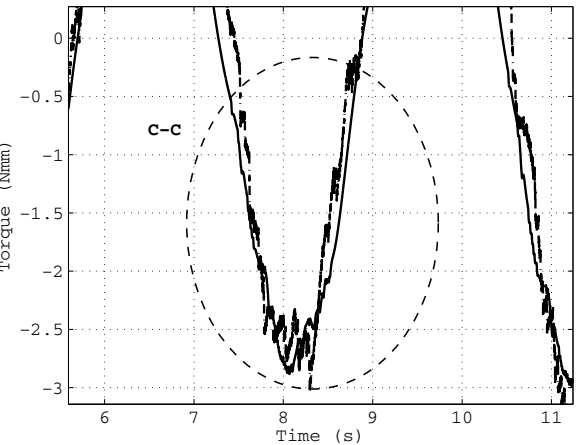

(b)

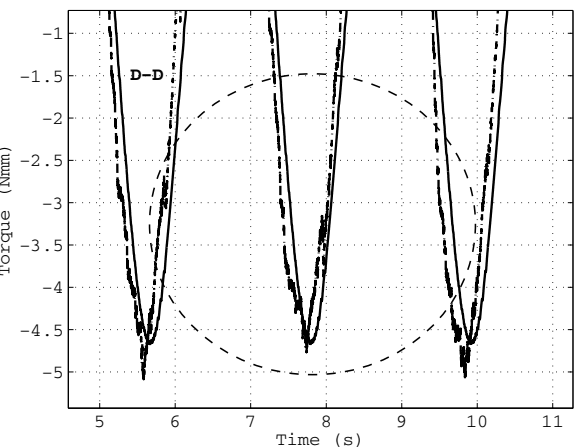

(d)

Fig. 14. Action reaction based force estimation experimental results

estimated interaction force to the actual one. The experimental values of the scaler observers gains ( $g_{\text {dist }}, g_{\text {reac }}$ and $\left.g_{f o}\right)$ are included in Table. 1 while the Luenberger-like state observer gain vector $(\mathrm{M})$ can be determined using the following characteristic equation

$$
\begin{gathered}
\left|s \mathrm{I}-(\mathrm{I}-c \mathrm{ML})^{-1}(\mathrm{~A}+k \mathrm{ML})\right|=\left(s-\mu_{1}\right) \ldots\left(s-\mu_{n}\right) \\
\mathrm{L}=\left[\begin{array}{lllll}
0 & 0 & 1 & 0 & \ldots
\end{array}\right]
\end{gathered}
$$

where $\left|s \mathrm{I}-(\mathrm{I}-c \mathrm{ML})^{-1}(\mathrm{~A}+k \mathrm{ML})\right|$ is the desired characteristic polynomial and $\mu_{1}, \ldots, \mu_{n}$ are the desired observer poles. The previous equation can be determined by subtracting the state space equation of the dynamical system from the action reaction state observer equation and further performing few algebraic manipulation to obtain a dynamical equation for the estimation error $(e=x-\widehat{x})$. The error dynamic therefore can be shown as

$$
\dot{\mathrm{e}}=(\mathrm{I}-c \mathrm{ML})^{-1}(\mathrm{~A}+k \mathrm{ML}) \mathrm{e}=\mathcal{A e}
$$


Here, the function $f($.$) is assumed to be a combination of a linear spring with stiffness k$ and linear energy dissipating element with viscous damping (c), $\mathrm{A} \in \mathbb{R}^{n \times n}$ is the system matrix, $\mathrm{I} \in \mathbb{R}^{n \times n}$ is the identity matrix and $\mathrm{L} \in \mathrm{R}^{1 \times n}$. The previous estimation error dynamical equation is not trivial, it is rather depending on the interaction force model between the dynamical subsystem and the input of the system, i.e., in driving the error dynamical equation, the interaction force model have to be determined and further used in the derivation of the estimation error dynamics.

The Luenberger-like state observer vector gain (M) can be determined by directly substituting the desired observer poles in (20). On the other hand, the scaler observer gains have to be selected in a way that satisfies two criterions, i.e., convergence time and noise attenuation since disturbance force, reaction force and the environmental interaction force a observer through low-pass filters. Therefore, the tradeoff between convergence time and noise attenuation restrict the selection of these scaler observers gains. It is worth noting that the action reaction force observer or state observer increases the flexibility and the range of these scaler gains since the noise associated with sensors is minimized as the number of measurements is reduced. The action reaction state observer or the action reaction force observers depends on a single measurement from the actuator along with the reference current input. Therefore, a single sensor has to be used in the realization of these state or force observers. The reference current signal is supplied through the computer control. Therefore, it is not a measurement it is rather a reference supplied signal. The pervious argument indicates that the action reaction force observer is depending on a single measurement from the dynamical system, the sensor noise is therefore reduced as the number of sensors utilized in the entire control system is minimized. It is worth noting that throughout the previous experiments the velocity measurement was obtained by differentiating the position encoder signal through a low-pass filter with cut-off frequency $g_{l}$, this explains the noise nature of the estimated interaction force depicted in Fig. 13 and Fig. 14. Therefore, it is recommended to obtain this velocity signal through an optimal filter such as Kalman filter rather than using a simple low-pass filter to attenuate the noise effect. Nevertheless, the results obtained in this chapter demonstrate the validity of the proposed sensorless force estimation techniques which can be further enhanced by utilizing optimal filter to reduce the sensor noise associated with the velocity measurement required to realize the action reaction state and force observers.

\section{Conclusion}

Sensorless force/torque control can be realized through utilization of the force observer techniques along with the action reaction state observer in order to provide an action reaction based force observer which can be used in the realization of sensorless force control. The action reaction based force observer does not require having any measurement from the dynamical system in contact with the environment except a single measurement from the input (actuator). The action reaction based force observer consists of four observers in cascade. The scalers and vector gains of these observers have to be selected such that convergence of the estimated interaction force to the actual one is guaranteed. The central idea of the action reaction state observer or the action reaction force observer is based on the realization of a natural feedback or a feedback-like force from the dynamical system which interact with the environment. This feedback-like force is then used in the design of a state and force observer by injecting the estimated reaction force onto the Luenberger-like state observer structure, 
thus allowing the estimated states or forces to converge to the actual ones through a stable error dynamics that can be accomplished by the proper selection of the observer scaler and vector gains. The action reaction based force servoing is introduced based on the action reaction state observer and the force observer. This force control technique is considered as a sensorless force control technique since force sensors are not utilized in the realization of the force control. In addition, velocity measurement of the non-collocated end effector in contact with the environment does not have to be measured. Experiments are conducted on a single input flexible lumped system with multi degree of freedom in contact with an environment with varying impedance. The estimation results of the states and the interaction forces were satisfactory showing fast convergence over a wide bandwidth of the dynamical system. Since the action reaction force observer depends on a single measurement from the system along with the reference current input, the associated noise problem is minimized and this allows the observer to work in a wide bandwidth. This in turn allows increasing the cut-off frequencies associated with each observer low-pass filter in order to reduce the convergence time of each observer of the action reaction state or force observer.

\section{References}

Huang, H., Sun, D., Mills, J. K. \& Li, W. J. (2007). Visual-based impedance force control of three-dimensional cell injection system, Proceedings of IEEEInt. Conf. on Robotics and Automation, ICRA'07, IEEE, Roma, pp. 4196-4201.

Iida, W. \& Ohnishi, K. (2003). Sensorless force control with force error observer, Proceedings of IEEEInt. Conf. on Industrial Technology, ICIT'11, IEEE, Maribor, pp. 157-162.

Karunakar, S. B. \& Goldenberg, A. A. (1988). Sensorless action-reaction-based residual vibration suppression for multi-degree-of-freedom flexible systems, Proceedings of IEEEInt. Symp. on Intelligent Control, ISIC'98, IEEE, Arlington, VA, pp. 412 - 417.

Katsura, S., Irie, K. \& Ohishi, K. (2008). Wideband force control by position-acceleration integrated disturbance observer, IEEE Trans. Ind Electron 55.(4.): 1699-1706.

Katsura, S., Matsumoto, Y. \& Ohnishi, K. (2007). Modeling of force sensing and validation of disturbance observer for force control, IEEE Trans. Ind Electron 54.(1.): 530-538.

Katsura, S. \& Ohnishi, K. (2007). Force servoing by flexible manipulator based on resonance ratio control, IEEE Trans. Ind Electron 54.(1.): 56-67.

Khalil, I. S. M. \& Sabanovic, A. (2010a). Action-reaction based parameters identification and states estimation of flexible systems, Proceedings of IEEEInt. Conf. Industrial Technology, ISIE'10, IEEE, PARI, pp. 46-51.

Khalil, I. S. M. \& Sabanovic, A. (2010b). Sensorless action-reaction-based residual vibration suppression for multi-degree-of-freedom flexible systems, Proceedings of IEEEInt. Conf. Industrial Technology, IECON'10, IEEE, Glendale, AZ, pp. 1633-1638.

Khalil, I. S. M. \& Sabanovic, A. (2011). A novel state observer for dynamical systems with inaccessible outputs, Proceedings of IEEEInt. Conf. Mechatronics, ICM'11, IEEE, Istanbul, pp. - .

Khalil, I. S. M. \& Sabanovic, A. (n.d.). Action reaction state observer for single input systems with compliance, IEEE Trans. Ind Electron .

Li, Y. F. \& Chen, X. B. (1998). On the dynamic behavior of a force/torque sensor for robots, IEEE Trans. Ind Electron 47.(1.): 304-308. 
Murakami, T., Yu, F. \& Ohnishi, K. (1993). Torque sensorless control in multidegree-of-freedom manipulator, IEEE Trans. Ind Electron 40.(2.): 259-265.

O'Connor, W. J. (1998). Position control of flexible robot arms using mechanical waves, ASME J. Dyn. Syst., Meas. Control 120.(3.): 334-339.

O'Connor, W. J. (2003). A gantry crane problem solved, ASME J. Dyn. Syst., Meas. Control 120.(4.): 569-576.

O'Connor, W. J. (2007a). Theory of wave analysis of lumped flexible systems, Proceedings of IEEEInt. Conf. American Control, ACC'07-NEW YORK, IEEE, NEW YORK, pp. 4215-4220.

O'Connor, W. J. (2007b). Wave-based analysis and control of lump-modeled flexible robots, IEEE Trans. Robotics 23.(.): 1552-3098.

Ohnishi, K., Shibata, M. \& Murakami, T. (1996). Motion control for advanced mechatronics, IEEE/ASME Trans. Mechatronics 1.(1.): 56-67.

Savia, M. \& Koivo, H. N. (2009). Contact micromanipulation-survey of strategies, IEEE/ASME Trans. Mechatronics 14.(4.): 504-514. 


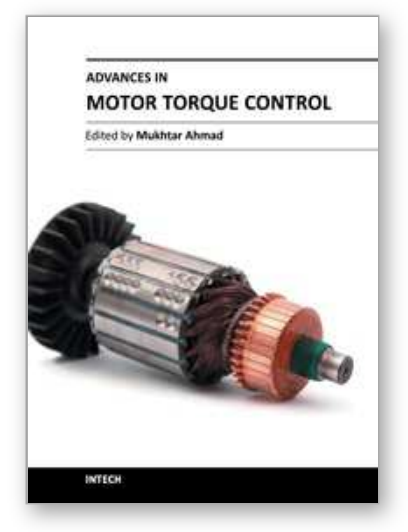

\author{
Advances in Motor Torque Control \\ Edited by Dr. Mukhtar Ahmad
}

ISBN 978-953-307-686-7

Hard cover, 144 pages

Publisher InTech

Published online 22, September, 2011

Published in print edition September, 2011

Electric motors are widely used in industries to convert electrical energy into mechanical form. Control techniques are designed to improve the performance and efficiency of the drive so that large amounts of electrical energy can be saved. This book is primarily written with the objective of providing necessary information on use of electric motors for various applications in industries. During the last ten years a number of methods of control of electric drives have emerged. Some of these methods are described in this book. The reader will be able to understand the new methods of control used in drives, e.g. direct and sensorless control. Also the application of motor control in dentistry, the effect of human reaction and improvement of the efficiency of drives with control have been described.

\title{
How to reference
}

In order to correctly reference this scholarly work, feel free to copy and paste the following:

Asif Sabanovic and Islam Khalil (2011). Sensorless Torque/Force Control, Advances in Motor Torque Control, Dr. Mukhtar Ahmad (Ed.), ISBN: 978-953-307-686-7, InTech, Available from:

http://www.intechopen.com/books/advances-in-motor-torque-control/sensorless-torque-control

\section{INTECH}

open science | open minds

\section{InTech Europe}

University Campus STeP Ri

Slavka Krautzeka 83/A

51000 Rijeka, Croatia

Phone: +385 (51) 770447

Fax: +385 (51) 686166

www.intechopen.com

\section{InTech China}

Unit 405, Office Block, Hotel Equatorial Shanghai

No.65, Yan An Road (West), Shanghai, 200040, China

中国上海市延安西路65号上海国际贵都大饭店办公楼 405 单元

Phone: +86-21-62489820

Fax: $+86-21-62489821$ 
(C) 2011 The Author(s). Licensee IntechOpen. This chapter is distributed under the terms of the Creative Commons Attribution-NonCommercialShareAlike-3.0 License, which permits use, distribution and reproduction for non-commercial purposes, provided the original is properly cited and derivative works building on this content are distributed under the same license. 